\title{
(6) OPEN ACCESS \\ Outcomes of ab interno trabeculectomy with the trabectome after failed trabeculectomy
}

\author{
I I Bussel, ${ }^{1}$ K Kaplowitz, ${ }^{2}$ J S Schuman,,${ }^{1,3}$ N A Loewen, ${ }^{1}$ Trabectome Study Group
}

'Department of Ophthalmology, UPMC Eye Center, Eye and Ear Institute, Ophthalmology and Visual Science Research Center, University of Pittsburgh School of Medicine, Pittsburgh, Pennsylvania, USA

${ }^{2}$ Department of Ophthalmology, Stony Brook University School of Medicine, Stony Brook, New York, USA

${ }^{3}$ Department of Bioengineering, Swanson School of Engineering, University of Pittsburgh, Pittsburgh, Pennsylvania, USA

\section{Correspondence to} Dr Nils A Loewen, Department of Ophthalmology, UPMC Eye Center, Eye and Ear Institute, Ophthalmology and Visual Science Research Center, University of Pittsburgh School of Medicine, 203 Lothrop St, \#819, Pittsburgh, PA 15235, USA, loewen.nils@gmail.com

Received 1 December 2013 Revised 8 July 2014 Accepted 26 July 2014 Published Online First 28 August 2014

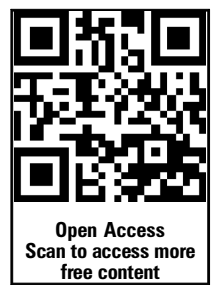

CrossMark

To cite: Bussel II, Kaplowitz K, Schuman JS, et al. Br J Ophthalmol 2015:99:258-262.

\section{ABSTRACT}

Aim To evaluate outcomes of ab interno trabeculectomy (AIT) with the trabectome following failed trabeculectomy.

Methods Prospective study of AlTs and phaco-AlTs after a failed trabeculectomy. The indication for AIT was intraocular pressure (IOP) above target on maximally tolerated therapy, and for phaco-AIT a visually significant cataract and need to lower IOP or glaucoma medications. Outcomes included IOP, medications, complications, secondary procedures and success, defined as IOP of less than $21 \mathrm{~mm} \mathrm{Hg}$ and a greater than $20 \%$ reduction from baseline without further surgery. Exclusion criteria were trabeculectomy less than 3 months prior to AIT or follow-up under 1 year. Results Seventy-three eyes of 73 patients with 1 year follow-up were identified. At 1 year, mean IOP in AIT significantly decreased by $28 \%$ from $23.7 \pm 5.5 \mathrm{~mm} \mathrm{Hg}$, and medications from $2.8 \pm 1.2$ to $2 \pm 1.3(n=58)$. In phaco-AIT, the mean IOP decreased $19 \%$ from 20 $\pm 5.9 \mathrm{~mm} \mathrm{Hg}$ and medications from $2.5 \pm 1.5$ to $1.6 \pm 1.4$ $(n=15)$. Transient hypotony occurred in $7 \%$, and further surgery was necessary in $18 \%$. For AlT and phaco-AIT, the 1 -year cumulative probability of success was $81 \%$ and $87 \%$, respectively.

Conclusions Both AIT and phaco-AIT showed a reduction in IOP and medication use after 1 year, suggesting that AlT with or without cataract surgery is a safe and effective option following failed trabeculectomy.

\section{INTRODUCTION}

Trabeculectomy, introduced in the $1960 \mathrm{~s}$ as an advancement from full thickness to guarded filtration, is still often called the standard of glaucoma surgery because it can achieve an average intraocular pressure (IOP) of $12.7 \pm 5.8 \mathrm{~mm} \mathrm{Hg}$ at 1 year, ${ }^{1}$ and an average of $13.5 \pm 5.3 \mathrm{~mm} \mathrm{Hg}$ in repeat trabeculectomy. ${ }^{2}$ It also has the highest complication rate of all current glaucoma treatment options with a rate of $39 \%$ early and $38 \%$ late potentially visionthreatening events adding up to a cumulative probability of $77 \%$ in a recent randomised controlled trial $^{3}$ that is consistent with prior findings of a $78 \%$ cumulative complication rate. ${ }^{4}$ Performing this procedure a second time is less effective than the initial trabeculectomy. ${ }^{5}$

$\mathrm{Ab}$ interno trabeculectomy (AIT) with the trabectome (Neomedix, Inc., Tustin, California) is different from cautery because, similar to disruptive or ablative ophthalmic lasers, this plasma-mediated ablation uses ionisation and disintegration of trabecular meshwork (TM) with highly confined heat dissipation: histology showed no thermal damage to adjacent tissues or the outer wall with the exception of rare coagulation effects at the margins of the immediate ablation area. ${ }^{6}$ AIT can lower IOP in most types of open-angle glaucoma with an intact downstream drainage system and do so with a favourable safety profile. ${ }^{7}$ Since it is performed through a clear-corneal incision, subsequent filtration surgery is not affected. ${ }^{8}$ The efficacy of AIT following fistulating surgery has not been examined because it is usually performed in patients with ocular hypertension or early forms of glaucoma, while trabeculectomy is deemed appropriate for more advanced stages. An additional concern was that AIT after traditional trabeculectomy might be ineffective because of atrophy after the conventional outflow tract is bypassed. ${ }^{9}$ Subendothelial TM deposits and an amorphous material in the juxtacanalicular tissue narrowing the lumen were noted in human eyes following trabeculectomy, with a significant correlation between lower IOPs and smaller canals. ${ }^{10}$ Until now, it was not known whether Schlemm's canal and collector sizes may be too small for AIT to be successful even after IOP increased again in eyes that had failed filtration surgery.

The purpose of this study was to evaluate 1-year outcomes of the few AIT surgeries performed either alone or combined with phacoemulsification in patients with a failed trabeculectomy. We hypothesised, that by removing the diseased meshwork and subendothelial plaques, conventional outflow might be restored to a level that allows patients to avoid another filtration surgery and, that removal of more diseased tissue in advanced glaucomas might result in a more pronounced IOP response than at early stages.

\section{METHODS}

Subjects were enrolled from the Trabectome Study Group database (previously described) ${ }^{711}$ with data relating to more than 5000 surgery outcomes, and divided into patients who had only AIT or AIT combined with phacoemulsification (phaco-AIT). AIT is a plasma surgery ablation technique that uses a bipolar $550 \mathrm{kHz}$ electrode tip to remove the TM, the primary resistance to outflow. Approximately $120-180^{\circ}$ of nasal TM was ablated. ${ }^{12}{ }^{13}$ The main incisions were made by right-handed surgeons at $220^{\circ}$ in right eyes and $20^{\circ}$ in left eyes. The same incision was used in phaco-AIT for both parts of the procedure. Data for this study was collected with institutional review board approval, in accordance with the Declaration of Helsinki and the Health Insurance Portability and Accountability Act.

This prospective interventional cohort included all patients with a diagnosis of glaucoma (with or without visually significant cataract), who had a failed trabeculectomy at least 3 months prior, and 
had at least 1 year of follow-up. Subjects were excluded if they had missing preoperative IOP data, end-stage visual field (VF) damage, or had AIT combined with any other procedure besides phacoemulsification.

The indication for AIT consisted of IOP above target with worsening glaucoma on maximally tolerated medical or laser therapy. The specific target IOP was set on a case-by-case basis by the individual treating physician as the maximum IOP estimated to prevent further nerve damage. The indication for phaco-AIT consisted of a visually significant cataract with at least $20 / 50$ on brightness acuity test and the need to lower IOP or the number of glaucoma medications. VF status of all patients was categorised as early, moderate, or advanced by individual glaucoma specialists based on the most recent Humphrey VF exams (Zeiss, Jena, Germany). When VFs could not be categorised, for instance, due to non-glaucomatous VF changes, or unreliable test performance by software assessment, exams were categorised as 'other' not to confound stratification. All patients had a comprehensive slit lamp and ophthalmoscopy exam prior to surgery.

We compared IOP measured by Goldmann applanation tonometry and the number of glaucoma medications within each group using the paired $t$ test. A finding of $p<0.05$ was considered statistically significant. All visual acuities were converted to logarithm of the minimum angle of resolution (logMar). Survival plots were created using Kaplan-Meier survival analysis. Success was defined as a final IOP of less than $21 \mathrm{~mm} \mathrm{Hg}$ and a greater than $20 \%$ reduction from baseline without further surgery. Scattergrams were used to illustrate the proportions of study subjects who met various criteria for success.

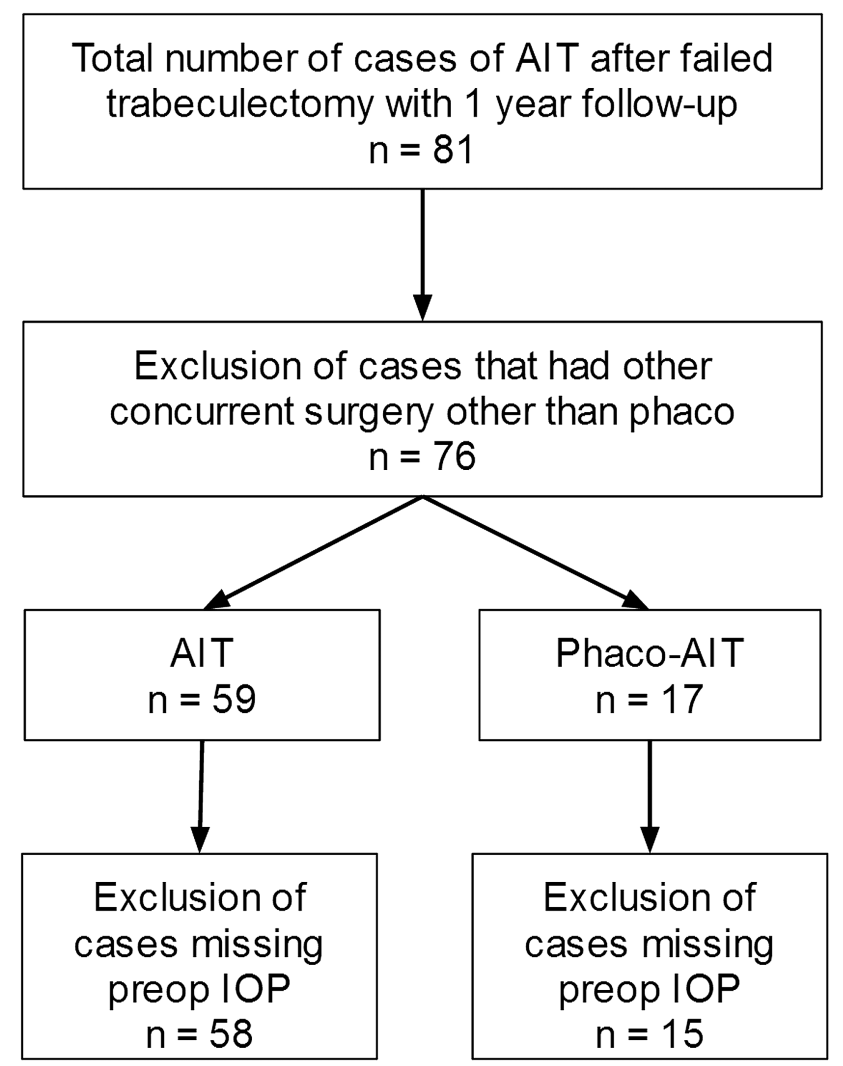

Figure 1 Trabectome Study Group Database. Cases analysed after accounting for exclusion criteria (AIT, ab interno trabeculectomy; phaco-AIT, phacoemulsification cataract surgery combined with AIT; IOP, intraocular pressure).
RESULTS

After applying the exclusion criteria, a total of 73 eyes in 73 patients were identified who were treated with AIT after failed trabeculectomy that were followed for a minimum of 1 year (figure 1). Baseline patient demographics are presented in table 1.

Figure 2 shows the preoperative and postoperative IOP measurements and change in glaucoma medications over time in AIT and phaco-AIT, respectively. In the AIT group, the mean IOP was reduced from $23.7 \pm 5.5$ to $16.2 \pm 3.9 \mathrm{~mm} \mathrm{Hg}(28 \%$ mean reduction, $\mathrm{p}<0.01$ ) and the number of medications was reduced from $2.8 \pm 1.2$ to $2.0 \pm 1.3(\mathrm{p}<0.01)$ at 1 year. In the phaco-AIT group, the mean IOP was reduced from $20.0 \pm 5.9$ to $15.6 \pm 5.1 \mathrm{~mm} \mathrm{Hg}(19 \%$ mean reduction, $\mathrm{p}=0.11)$, while the

Table 1 Baseline patient demographics

\begin{tabular}{|c|c|c|}
\hline & $\begin{array}{l}\text { AIT } \\
(n=58)\end{array}$ & $\begin{array}{l}\text { Phaco-AIT } \\
(n=15)\end{array}$ \\
\hline \multicolumn{3}{|l|}{ Age } \\
\hline Mean \pm SD & $68 \pm 17$ & $71 \pm 10$ \\
\hline Range & $16-90$ & $57-85$ \\
\hline \multicolumn{3}{|l|}{ Gender, n (\%) } \\
\hline Female & $28(48)$ & 7 (47) \\
\hline Male & $28(48)$ & $8(53)$ \\
\hline NR & $2(3)$ & $0(0)$ \\
\hline \multicolumn{3}{|l|}{ Race, n (\%) } \\
\hline African-American & $3(5)$ & $0(0)$ \\
\hline Asian & $3(5)$ & $1(7)$ \\
\hline Caucasian & $46(79)$ & $10(67)$ \\
\hline Hispanic & $3(5)$ & $2(13)$ \\
\hline Others & $3(5)$ & $2(13)$ \\
\hline \multicolumn{3}{|l|}{ Diagnosis, n (\%) } \\
\hline POAG & $49(84)$ & $11(73)$ \\
\hline Pseudoexfoliation glaucoma & $4(7)$ & $2(13)$ \\
\hline Juvenile & $1(2)$ & $0(0)$ \\
\hline CACG & $1(2)$ & $0(0)$ \\
\hline Pigment dispersion & $1(2)$ & $1(7)$ \\
\hline Uveitic glaucoma & $1(2)$ & $1(7)$ \\
\hline Others & $1(2)$ & $0(0)$ \\
\hline \multicolumn{3}{|l|}{ Preoperative Snellen acuity, n (\%) } \\
\hline $\log M a r(m e a n \pm S D)$ & $0.39 \pm 0.49$ & $0.51 \pm 0.28$ * \\
\hline \multicolumn{3}{|l|}{ Postoperative Snellen acuity, n (\%) } \\
\hline $\log M a r(m e a n \pm S D)$ & $0.48 \pm 0.71$ & $0.14 \pm 0.13$ \\
\hline \multicolumn{3}{|l|}{$V F, n(\%)$} \\
\hline Mild & $6(10)$ & $2(13)$ \\
\hline Moderate & $14(24)$ & $2(13)$ \\
\hline Advanced & $24(41)$ & $4(27)$ \\
\hline Others & $14(24)$ & $7(47)$ \\
\hline \multicolumn{3}{|l|}{ Disc C/D, n (\%) } \\
\hline$<0.7$ & $3(5)$ & $2(13)$ \\
\hline $0.7-0.8$ & $17(29)$ & $5(33)$ \\
\hline$>0.8$ & $30(52)$ & $6(40)$ \\
\hline NR & $8(14)$ & $2(13)$ \\
\hline \multicolumn{3}{|l|}{ Shaffer grade, n (\%) } \\
\hline 1 & $0(0)$ & $0(0)$ \\
\hline II & $4(7)$ & $0(0)$ \\
\hline III & $8(14)$ & $6(40)$ \\
\hline IV & $31(53)$ & $4(27)$ \\
\hline NR & $15(26)$ & $5(33)$ \\
\hline
\end{tabular}


AIT

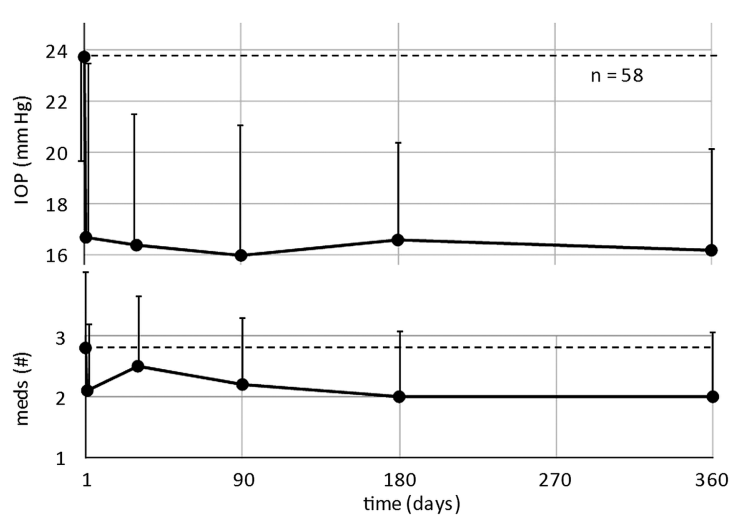

phaco-AIT

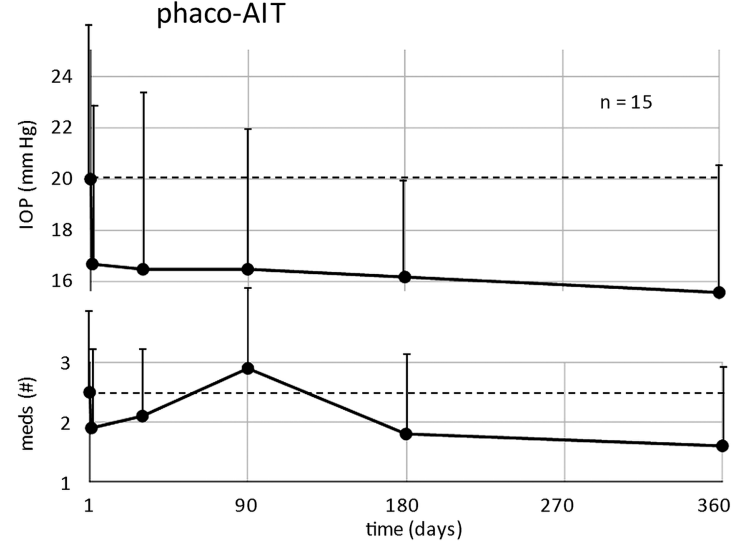

Figure 2 Preoperative and postoperative intraocular pressure (IOP) measurements (top) and change in glaucoma medications (bottom) over time in ab interno trabeculectomy (AIT) (left) and phaco-AIT (right).

mean number of medications was reduced from $2.5 \pm 1.5$ to 1.6 $\pm 1.4(\mathrm{p}=0.24)$ at 1 year.

Stratifying all cases by VF status (figure 3), subjects with mild or moderate VF status had a mean baseline IOP of 24.1 $\pm 5.3 \mathrm{~mm} \mathrm{Hg}$ which was reduced to $17.1 \pm 4 \mathrm{~mm} \mathrm{Hg}$ at 1 year ( $24 \%$ reduction, $\mathrm{p}<0.01)$. The mean number of glaucoma medications was reduced from $2.2 \pm 1.2$ to $1.8 \pm 1.2$ at 1 year $(p=1.0)$. Patients with advanced VF status had a mean baseline IOP of $22.9 \pm 5 \mathrm{~mm} \mathrm{Hg}$ which was reduced to $15 \pm 4.3 \mathrm{~mm} \mathrm{Hg}$ at 1 year (31\% reduction, $\mathrm{p}<0.01)$. The mean number of glaucoma medications was reduced from $3 \pm 1.2$ to $2 \pm 1.4$ at 1 year $(p=0.04)$.

Figure 4 shows the survival plots for AIT and phaco-AIT. For AIT and phaco-AIT, the 1-year cumulative probability of success was $81 \%$ and $87 \%$, respectively. Table 2 presents success proportions by various success criteria, and figure 5 shows scattergrams that allow for visualisation of individual outcomes at 12 months that are captured by those criteria.

Table 3 presents secondary procedures and complications. A total of 13 cases $(18 \%)$ went on to require further glaucoma surgery within 1 year. Five subjects $(7 \%)$ had transient hypotony $(\mathrm{IOP}<5)$, but in all these cases, the hypotony spontaneously resolved and there was no loss of vision. There were no reported cases of aqueous misdirection, infection, wound leaks, choroidal effusion, or suprachoroidal haemorrhage.

\section{DISCUSSION}

Trabeculectomy has long been considered the standard for surgical treatment of glaucoma. Recently, epibulbar glaucoma drainage devices have been shown to be similarly effective. ${ }^{3}$

In this study, we evaluated for the first time the outcomes of AIT and phaco-AIT in subjects with glaucoma who had previously failed trabeculectomy. Such minimally invasive glaucoma procedures had been hypothesised to be ineffective after outflow tract bypassing surgery, and generally thought to be less effective than traditional glaucoma drainage procedures. We found that after 1 year, AIT performed alone showed a statistically significant decrease in IOP and in the number of glaucoma medications, while phaco-AIT resulted in a similar trend but did not reach statistical significance for medications (given difference of means and SD observed, the sample size would have to be at least 20). Furthermore, the effect was similar when stratified by VF status. Our results suggest that AIT may offer an attractive therapeutic option for patients who require further IOP lowering after failed glaucoma surgery, and especially in those who are unwilling or unable to tolerate repeat traditional glaucoma surgery.

In this study, the mean IOP decreased by $28 \%$ from a baseline of $23.7 \mathrm{~mm} \mathrm{Hg}$ after AIT, and by $19 \%$ from a baseline of
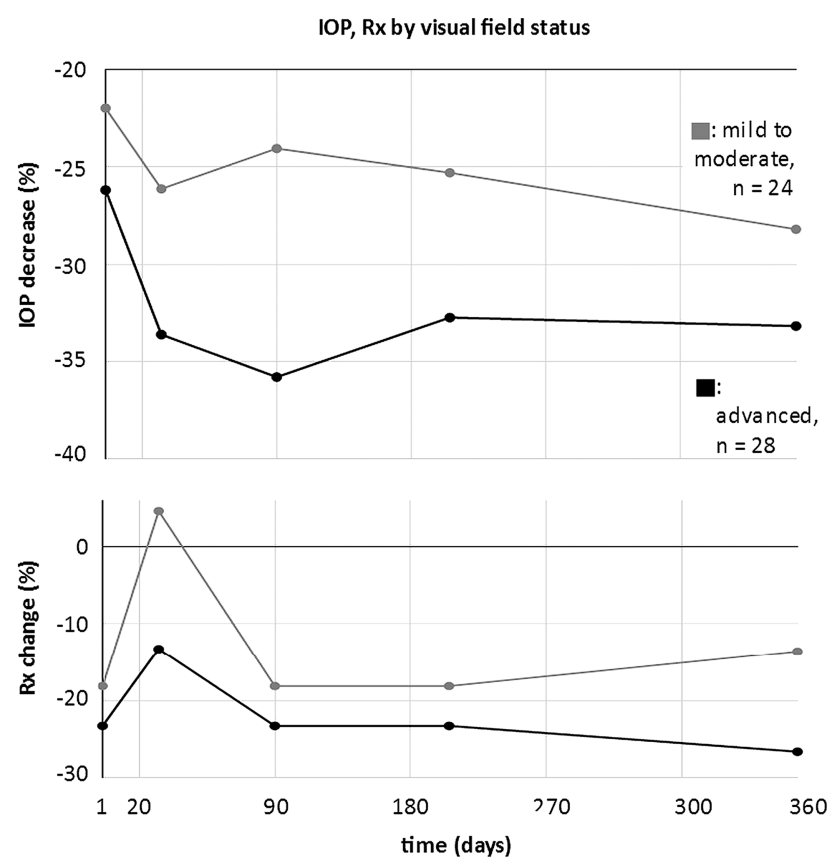

\begin{tabular}{c|ccccc} 
time, days & 1 & 30 & 90 & 180 & 360 \\
\hline $\begin{array}{c}\text { IOP change, \% (SD) } \\
\text { mild to moderate }\end{array}$ & $-22(42)$ & $-26(38)$ & $-24(33)$ & $-25(26)$ & $-28(29)$ \\
$\begin{array}{c}\text { IOP change, \% (SD) } \\
\text { advanced }\end{array}$ & $-26(37)$ & $-34(26)$ & $-36(29)$ & $-33(31)$ & $-33(29)$ \\
\hline $\begin{array}{c}\text { Rx change, \% (SD) } \\
\text { mild to moderate }\end{array}$ & $-18(73)$ & $5(73)$ & $-18(50)$ & $-18(59)$ & $-14(50)$ \\
$\begin{array}{c}\text { Rx change, \% (SD) } \\
\text { advanced }\end{array}$ & $-23(63)$ & $-13(37)$ & $-23(43)$ & $-23(33)$ & $-27(43)$
\end{tabular}

Figure 3 Relative decrease of intraocular pressure (IOP) (top) and number of topical glaucoma medications (bottom graph) in patients with mild to moderate (gray) and advanced (black). Patients with advanced visual field loss had a larger IOP decrease on average. Bottom: table with means and SDs (all $p>0.05$ ). 
AIT survival plot

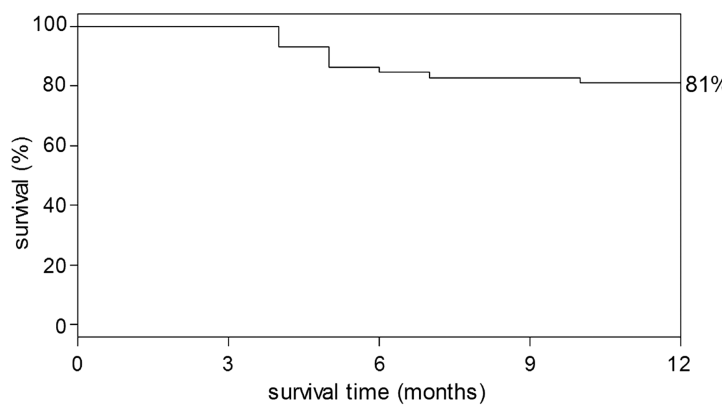

phaco-AIT survival plot

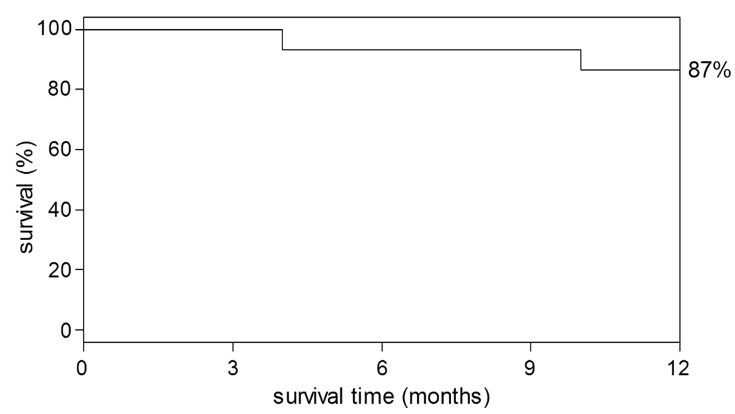

Figure 4 Kaplan-Meier survival plots for ab interno trabeculectomy (AIT) (left) and phacoemulsification cataract surgery combined with AIT (phaco-AIT) (right) with success defined as a final intraocular pressure of less than $21 \mathrm{~mm} \mathrm{Hg}$ and a greater than $20 \%$ reduction from baseline without further surgery.

$20 \mathrm{~mm} \mathrm{Hg}$ after phaco-AIT, which matches previous reports that also report a higher baseline IOP in the AIT group. ${ }^{14}{ }^{15}$ In common with those studies, and reflecting different indications, IOP reduction was greater in AIT alone compared with the phaco-AIT group. Francis and colleagues reported a 1-year phaco-AIT case series of 304 subjects with an overall success

Table 2 Success proportions by various criteria

\begin{tabular}{|c|c|c|}
\hline & AIT n (\%) & Phaco-AIT n (\%) \\
\hline Total number of survived cases & 47 & 13 \\
\hline (1) $<21 \mathrm{~mm} \mathrm{Hg}$ or $20 \%$ IOP reduction & $44(94)$ & $12(92)$ \\
\hline (2) $<18 \mathrm{~mm} \mathrm{Hg}$ or $20 \%$ IOP reduction & $37(79)$ & $11(85)$ \\
\hline (3) $<15 \mathrm{~mm} \mathrm{Hg}$ or $20 \%$ IOP reduction & $33(70)$ & $10(77)$ \\
\hline (4) $<12 \mathrm{~mm} \mathrm{Hg}$ or $20 \%$ IOP reduction & $31(66)$ & $8(62)$ \\
\hline (5) $20 \%$ IOP reduction & $31(66)$ & $8(62)$ \\
\hline (6) $<21 \mathrm{~mm} \mathrm{Hg}$ & $42(89)$ & $12(92)$ \\
\hline (7) $<18 \mathrm{~mm} \mathrm{Hg}$ & $31(66)$ & $10(77)$ \\
\hline (8) $<15 \mathrm{~mm} \mathrm{Hg}$ & $18(38)$ & $6(46)$ \\
\hline (9) $<12 \mathrm{~mm} \mathrm{Hg}$ & $6(13)$ & $2(15)$ \\
\hline (10) $<21 \mathrm{~mm} \mathrm{Hg}$ and $20 \%$ IOP reduction & $29(62)$ & $8(62)$ \\
\hline (11) $<18 \mathrm{~mm} \mathrm{Hg}$ and $20 \%$ IOP reduction & $25(53)$ & $7(54)$ \\
\hline (12) $<15 \mathrm{~mm} \mathrm{Hg}$ and $20 \%$ IOP reduction & $16(34)$ & $4(31)$ \\
\hline (13) $<12 \mathrm{~mm} \mathrm{Hg}$ and $20 \%$ IOP reduction & $6(13)$ & $2(15)$ \\
\hline
\end{tabular}

rate of $64 \%$, defining success as $\geq 20 \%$ drop in IOP, or a decrease in glaucoma medications without the need for additional medications or glaucoma procedures, including laser trabeculoplasty. ${ }^{15}$ Olali reported an $82 \%$ success (defined as $\mathrm{IOP} \leq 21 \mathrm{~mm} \mathrm{Hg}$ ) 1 year after a repeat trabeculectomy with mitomycin-C following a failed trabeculectomy. ${ }^{2}$ In our study, $89 \%$ of AIT and $92 \%$ of phaco-AIT patients had an IOP below $21 \mathrm{~mm} \mathrm{Hg}$ at 12 months. Although surgical successes cannot be compared directly, it is possible that patients who are phenotypically more likely to fail trabeculectomy due to fibrosis will fail the same procedure again from the same cause, while fibrosis may not play a primary role in ab interno trabeculectomy.

When conventional outflow is decreased following trabeculectomy, ${ }^{9}$ the TM develops subendothelial deposits. ${ }^{10}$ The findings reported here suggest that the distal outflow tract does not irreversibly atrophy, and that natural outflow can be restored. When stratifying results by baseline VF status as a surrogate for glaucoma status, IOP was lower in the group with more advanced disease, suggesting that removal of a more diseased outflow resistance can result in a larger decrease in IOP.

Our study had several limitations, including a potential selection bias towards worse outcomes, as only the initial 20 cases are required to be submitted to the Trabectome Study Group Database to obtain full certification followed by subsequent voluntary reporting. Though this was a prospective cohort, it is based on a limited sample size with a relatively short-term follow-up.
AIT

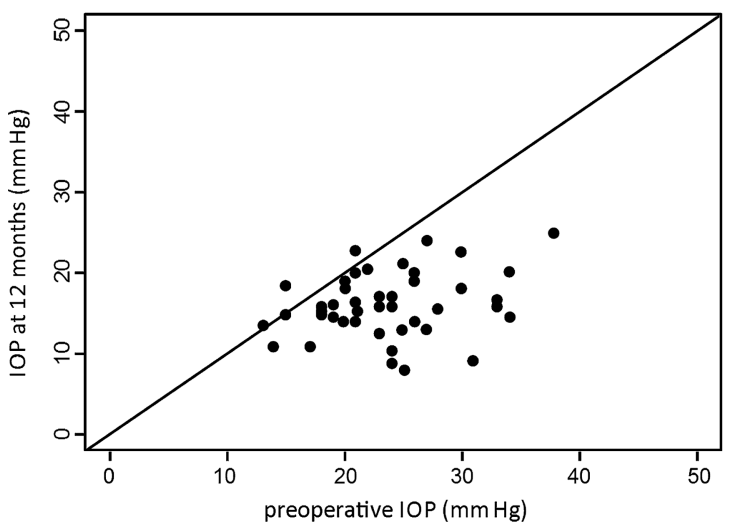

phaco-AIT

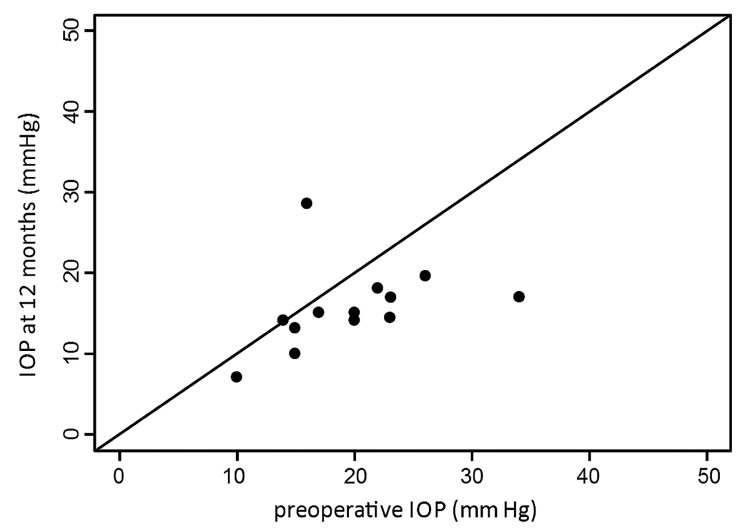

Figure 5 Scattergrams for ab interno trabeculectomy (AIT) (left) and phacoemulsification cataract surgery combined with AIT (phaco-AIT) (right) demonstrating intraocular pressure (IOP) change from preoperative baseline to final measurement at 12 months. 
Table 3 Secondary surgeries and postoperative complications

\begin{tabular}{lll}
\hline & AIT $\mathbf{n}(\%)$ & Phaco-AIT $\mathbf{n}(\%)$ \\
\hline Failures requiring further glaucoma surgery & & \\
Trabeculectomy & $2(3)$ & $2(13)$ \\
Aqueous shunt & $6(10)$ & $0(0)$ \\
Cyclophotocoagulation & $2(3)$ & $0(0)$ \\
Repeat AIT & $1(2)$ & $0(0)$ \\
Total secondary surgery & $11(19)$ & $2(13)$ \\
Complications & & \\
Transient hypotony (IOP $<5 \mathrm{~mm} \mathrm{Hg})$ & $3(5)$ & $2(14)$ \\
Aqueous misdirection & $0(0)$ & $0(0)$ \\
Infection & $0(0)$ & $0(0)$ \\
Wound leaks & $0(0)$ & $0(0)$ \\
Pain & $0(0)$ & $0(0)$ \\
Choroidal effusion & $0(0)$ & $0(0)$ \\
Choroidal haemorrhage & $0(0)$ & $0(0)$ \\
\hline AlT, ab interno trabeculectomy; IOP, intraocular pressure. &
\end{tabular}

In conclusion, AIT can be considered a viable therapeutic option for patients with a history of previously failed trabeculectomy who require further IOP lowering and are unwilling or unable to tolerate conventional glaucoma surgery.

Contributors IIB, KK, NAL and JSS contributed equally to data analysis and manuscript writing. NAL designed the study.

Competing interests NAL is a trabectome wetlab instructor.

Ethics approval Institutional review board.

Provenance and peer review Not commissioned; externally peer reviewed.

Open Access This is an Open Access article distributed in accordance with the Creative Commons Attribution Non Commercial (CC BY-NC 4.0) license, which permits others to distribute, remix, adapt, build upon this work non-commercially, and license their derivative works on different terms, provided the original work is properly cited and the use is non-commercial. See: http://creativecommons.org/ licenses/by-nc/4.0/

\section{REFERENCES}

1 Gedde SJ, Schiffman JC, Feuer WJ, et al. Treatment outcomes in the tube versus trabeculectomy study after one year of follow-up. Am J Ophthalmol 2007; 143:9-22.

2 Olali C, Rotchford AP, King AJ. Outcome of repeat trabeculectomies. Clin Experiment Ophthalmol 2011;39:658-64.

3 Gedde SJ, Schiffman JC, Feuer WJ, et al. Treatment outcomes in the Tube Versus Trabeculectomy (TVT) study after five years of follow-up. Am J Ophthalmol 2012;153:789-803.e2.

4 Bindlish R, Condon GP, Schlosser JD, et al. Efficacy and safety of mitomycin-C in primary trabeculectomy: five-year follow-up. Ophthalmology 2002;109:1336-41; discussion 1341-2.

5 Law SK, Shih K, Tran DH, et al. Long-term outcomes of repeat vs initial trabeculectomy in open-angle glaucoma. Am J Ophthalmol 2009;148:685-95.e1.

6 Francis BA, See RF, Rao NA, et al. Ab interno trabeculectomy: development of a novel device (Trabectome) and surgery for open-angle glaucoma. J Glaucoma 2006;15:68-73.

7 Ting JLM, Damji KF, Stiles MC, et al. Ab interno trabeculectomy: outcomes in exfoliation versus primary open-angle glaucoma. J Cataract Refract Surg 2012;38:315-23.

8 Jea SY, Mosaed S, Vold SD, et al. Effect of a failed trabectome on subsequent trabeculectomy. J Glaucoma 2012;21:71-5.

9 Lütjen-Drecoll E. Functional morphology of the trabecular meshwork in primate eyes. Prog Retin Eye Res 1999;18:91-119.

10 Johnson DH, Matsumoto Y. Schlemm's canal becomes smaller after successful filtration surgery. Arch Ophthalmol 2000;118:1251-6.

11 Minckler D, Mosaed S, Dustin L, et al. Trabectome (trabeculectomy-internal approach): additional experience and extended follow-up. Trans Am Ophthalmol Soc 2008;106:149-60.

12 Kaplowitz K, Schuman JS, Loewen NA. Techniques and outcomes of minimally invasive trabecular ablation and bypass surgery. Br J Ophthalmol 2014;98:579-85.

13 Kaplowitz K, Loewen NA. Minimally invasive glaucoma surgery: trabeculectomy ab interno. In: JR Samples, IIK Ahmed, Eds. Surgical innovations in glaucoma. New York: Springer, 2014:175-86.

14 Minckler DS, Baerveldt G, Alfaro MR, et al. Clinical results with the Trabectome for treatment of open-angle glaucoma. Ophthalmology 2005;112:962-7.

15 Francis BA, Minckler D, Dustin L, et al. Combined cataract extraction and trabeculotomy by the internal approach for coexisting cataract and open-angle glaucoma: initial results. J Cataract Refract Surg 2008;34:1096-103. 